\title{
Effective temperatures and lithium abundances of halo turnoff stars $\dagger$
}

\author{
P.E. Nissen ${ }^{1}$, C. Akerman ${ }^{2}$, M. Asplund ${ }^{3}$, \\ D. Fabbian ${ }^{3}$ and M. Pettini ${ }^{2}$ \\ ${ }^{1}$ Department of Physics and Astronomy, University of Aarhus, Denmark \\ email: pen@phys.au.dk \\ ${ }^{2}$ Institute of Astronomy, Cambridge, UK \\ ${ }^{3}$ Mount Stromlo Observatory, Australian National University, Australia
}

\begin{abstract}
Effective temperatures of 30 turnoff stars with $-3.2<[\mathrm{Fe} / \mathrm{H}]<-1.5$ have been derived from the profiles of Balmer lines in high S/N, VLT/UVES spectra. While the systematic error of $T_{\text {eff }}$ may be of the order of $100 \mathrm{~K}$, the differential values of $T_{\text {eff }}$ are determined with a one-sigma precision of $\sim 25 \mathrm{~K}$. These precise $T_{\text {eff }}$ values are used in a study of the slope and dispersion of the $\mathrm{Li}$ abundance as a function of $[\mathrm{Fe} / \mathrm{H}]$. A small, but significant cosmic dispersion in $A(\mathrm{Li})$ appears to be present exemplified by the two very metal-poor stars G64-12 and G64-37.
\end{abstract}

Keywords. Stars: fundamental parameters, stars: abundances, cosmology: early universe

\section{Introduction}

From the baryon-to-photon ratio determined from WMAP data in combination with SBBN calculations, the primordial ${ }^{7} \mathrm{Li}$ abundance is predicted to be ${ }^{7} \mathrm{Li} / \mathrm{H} \simeq 4 \times 10^{-10}$, which is a factor 3-4 higher than Li abundances found in metal-poor stars on the Spite plateau (Spite \& Spite 1982). The reason could be that the atmospheric Li abundance of these stars has been depleted, but models have difficulties in explaining the small dispersion in $\mathrm{A}(\mathrm{Li}) \equiv \log (\mathrm{Li} / \mathrm{H})+12.0$ among the plateau stars. Ryan et al. (1999), in particular, found a very small dispersion, $\sigma(A(\mathrm{Li}))=0.031 \mathrm{dex}$, around a metallicity trend, $\Delta A(\mathrm{Li}) / \Delta[\mathrm{Fe} / \mathrm{H}] \simeq 0.12$, for a sample of 21 turnoff stars with low metallicities. As a further study of this problem we have derived $\mathrm{Li}$ abundances for a sample of 30 metal-poor turnoff stars from VLT/UVES echelle spectra using effective temperatures derived from the profiles of Balmer lines.

\section{Effective temperatures and abundances}

The majority of the spectra used are from the C/O study of Akerman et al. (2004). Recently, the sample has been extended with new UVES spectra for 12 very metal-poor stars (unpublished). All these spectra have $R \simeq 60000$ and $S / N \simeq 300$, and $\mathrm{H} \beta$ was used to determine $T_{\text {eff }}$. In addition, we have included the more metal-poor stars $([\mathrm{Fe} / \mathrm{H}]<$ -1.5) from the ${ }^{6} \mathrm{Li}$ survey of Asplund et al. (2005). For these stars, $\mathrm{H} \alpha$ was used to derive $T_{\text {eff. }}$.

Theoretical profiles of the Balmer lines were calculated as described in Barklem et al. (2002) and used to calibrate an index measuring the flux in two bands in the line wings relative to the flux in two bands near the line center as a function of $T_{\text {eff }}$. By defining

$\dagger$ Based on observations collected at ESO, Chile (ESO No. 67.D-0106 and 73.D-0024) 

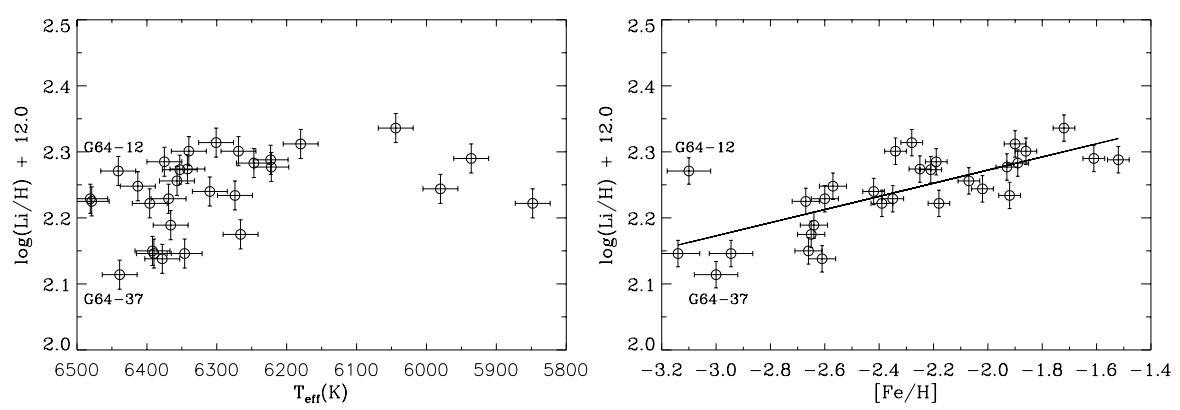

Figure 1. Li abundances vs. $T_{\text {eff }}$ and $[\mathrm{Fe} / \mathrm{H}]$

the $T_{\text {eff }}$ indices of the Balmer lines in this way, we avoid referring to the true continuum, which cannot be determined with high accuracy for echelle spectra.

The internal precision of the $T_{\text {eff }}$ determination appears to be very high. Effective temperatures derived from the left and the right wing of the $\mathrm{H} \beta$ line have a mean difference of $13 \pm 6 \mathrm{~K}$ only, and $T_{\text {eff }}$ 's determined from spectra of a given star obtained on different nights differ by less than $20 \mathrm{~K}$. For nine stars with both $\mathrm{H} \alpha$ and $\mathrm{H} \beta$ observations, there is an average difference $T_{\text {eff }}(\mathrm{H} \beta)-T_{\text {eff }}(\mathrm{H} \alpha)$ of $57 \mathrm{~K}$, but the rms dispersion of the difference is $\pm 19 \mathrm{~K}$ only. This suggests that the errors of differences in effective temperatures are very small, probably less than $25 \mathrm{~K}$. Systematic errors in $T_{\text {eff }}$ may, however, be considerably larger.

$[\mathrm{Fe} / \mathrm{H}]$ is determined from Fe II lines as described in Nissen et al. (2004). The Li abundances are derived from the Li I $6708 \AA$ line using MARCS model atmospheres and assuming LTE. From the error of the measured equivalent width of the Li line $( \pm 0.5 \mathrm{~m} \AA)$, and an internal error $\sigma\left(T_{\text {eff }}\right)= \pm 25 \mathrm{~K}$, we arrive at an expected rms precision of $\sigma(A(\mathrm{Li}))$ $=0.022$ dex for the differential values of the Li abundances.

\section{Results}

The derived values of $A(\mathrm{Li})$ are plotted as a function of $T_{\text {eff }}$ and $[\mathrm{Fe} / \mathrm{H}]$ in Fig. 1 . As seen there is hardly any significant trend with $T_{\text {eff }}$ but a probable increase of the Li abundance with $[\mathrm{Fe} / \mathrm{H}]$. A linear fit to the data gives $A(\mathrm{Li})=0.10[\mathrm{Fe} / \mathrm{H}]+2.47$ with a rms dispersion of 0.041 . This is significantly higher than the expected dispersion of 0.022 dex. A large contribution to the dispersion comes from the pair of very metal-poor stars, G64-12 and G64-37, which have nearly identical $\mathrm{H} \beta$ profiles corresponding to $T_{\text {eff }}=$ $6440 \mathrm{~K}$, but quite different values of the equivalent width of the Li I $6708 \AA$ line, 22.5 and $16.1 \mathrm{m \AA}$, respectively. Even without G64-12, the dispersion (0.035 dex) remains higher than expected. Hence, in contrast to the study of Ryan et al. (1999), our data point to a small, but significant cosmic dispersion in $A(\mathrm{Li})$ among metal-poor turnoff stars. This suggests that Li depletion has occurred in some of the Spite plateau stars.

\section{References}

Akerman, C.J., Carigi, L., Nissen, P.E., Pettini, M. \& Asplund M. 2004, A\&\&A 414, 931 Asplund, M., Lambert, D.L., Nissen, P.E., Primas, F. \& Smith V.V. 2005, in preparation Barklem, P.S., Stempels, H.C., Allende Prieto, C., Kochukhov, O.P., Piskunov, N. \& O'Mara, B.J. 2002, A\&A 385, 951

Nissen, P.E., Chen, Y.Q., Asplund, M. \& Pettini, M. 2004, A\&̈A 415, 993

Ryan, S.G., Norris, J.E., \& Beers, T.C. 1999, ApJ 523, 654

Spite, F. \& Spite, M. 1982, A\&A 115, 357. 